\title{
Intelligent Bus Stops in the Flexible Bus Systems
}

\author{
Razi Iqbal* and Muhammad Usman Ghani
}

Al-Khawarizmi Institute of Computer Science, University of Engineering and Technology, Lahore, Pakistan

Received 7 May 2013; Accepted 27 September 2014

\begin{abstract}
The purpose of this paper is to discuss Intelligent Bus Stops in a special Demand Responsive Transit (DRT), the Flexible Bus System. These Intelligent Bus Stops are more efficient and information rich than Traditional Bus Stops. The real time synchronization of the Flexible Bus System makes it unique as compared to Traditional Bus Systems. The Main concern is to make Bus Stops intelligent and information rich. Buses are informed about the no. of passengers waiting at the upcoming Bus Stops. If there are no passengers to ride or get off on upcoming Bus Stop, the Bus can skip that Bus Stop and head towards the next Bus Stop where passenger is waiting, which will decrease the ride time of the passengers on the Bus and also the wait time of the passengers waiting on the upcoming Bus Stops. Providing more information at Bus Stops about the Destination (Time to Destination, Distance to Destination etc.) and Buses (Bus Location, Arrival Time of Bus etc.) makes it easier for the passengers to decide whether to ride a particular Bus or not.
\end{abstract}

Keywords: Intelligent Transportation Systems, Demand Responsive Transits, Wireless communication, ZigBee

\section{Introduction}

The main objective of this paper is to do a research on Bus Stops in public transportation system to make them intelligent and easy to use. The Intelligent Bus Stops are the part of an efficient public Transportation System; we call it "The Flexible Bus System" (FBS). The word "Flexible" is used in terms of Bus routes and customer demands, since in FBS, Buses can change their routes according to Passenger demands. FBS is a Demand Responsive Transit (DRT). DRT systems reduce the wait time and the ride time of the passengers by using the information provided by the passengers about their source and the destination. There are already lots of Demand Responsive Transit (DRT) systems which are better than Traditional Bus Systems but the Flexible Bus System makes this system an ease of use for the passengers by supplying more and more information at the Bus Stops and an efficient algorithm reduces the wait time of the passengers at the Bus Stops. FBS replaces the scheduled bus lines in a way that buses can dynamically change their routes according to passenger's demands. By informing the passengers about the accurate bus locations makes this system much more efficient and information rich as compared to Traditional Demand Responsive Transits. The Flexible Bus System can serve as a search engine for the tourists or one-time riders and guides them about the nearby famous places, restaurants, hospitals and etc. System is intelligent in a sense as well that it will display famous places, restaurant, hospitals and etc. corresponding to each Bus Stop. The graphical user interface is very easy to use and designed in such a way that it lets users choose from the

\footnotetext{
*E-mail address: razi.iqbal@kics.edu.pk

ISSN: 1791-2377 @ 2014 Kavala Institute of Technology. All rights reserved.
}

given choices instead of making them type in most of the cases. The Systems installed at Intelligent Bus Stops are very speedy and efficient in a sense that they respond to Passenger's demands instantly just like Automated Teller Machines (ATMs) at Bank. The Ticketing System of Flexible Bus System is also automated for registered members; the passengers don't have to carry money for travelling by Flexible Bus System. Each time they travel the corresponding charges are deducted from their Bank accounts registered with Flexible Bus System. One time riders or tourists still have to pay by cash.

\section{Research Originality}

The Intelligent Bus Stops in Flexible Bus Systems are an effort to make Bus Stops more passenger friendly. Passengers are informed about the real time location of the buses which makes it easy for the passengers to decide whether to ride a particular bus or not. It also suggests which Bus a passenger should ride and how long the Bus will take to reach the Bus Stop of the waiting passenger. Similarly it also shows the expected arrival time at the destination. The System is very efficient and inexpensive in a sense that Buses are made to dynamically change the routes by informing them about the no. of passengers waiting on the upcoming bus stops so that Buses will drive to the Bus Stops where passengers are waiting instead of driving to the Bus Stops where there are no passengers, which will definitely reduce the fuel cost and hence the total cost of the System. The main concern of this research is to make a Bus System an ease of use for the passengers, they don't have to wait for buses on the bus stops for long and the passengers are also aware of the wait time (how much time 
they have to wait for a particular bus on the Bus Stop?). The system is totally automated which makes this system speedy.

\section{Proposed Model For Flexible Bus Systems}

Figure 1 shows our proposed model of the Flexible Bus System. There is a Control Centre which is the central part

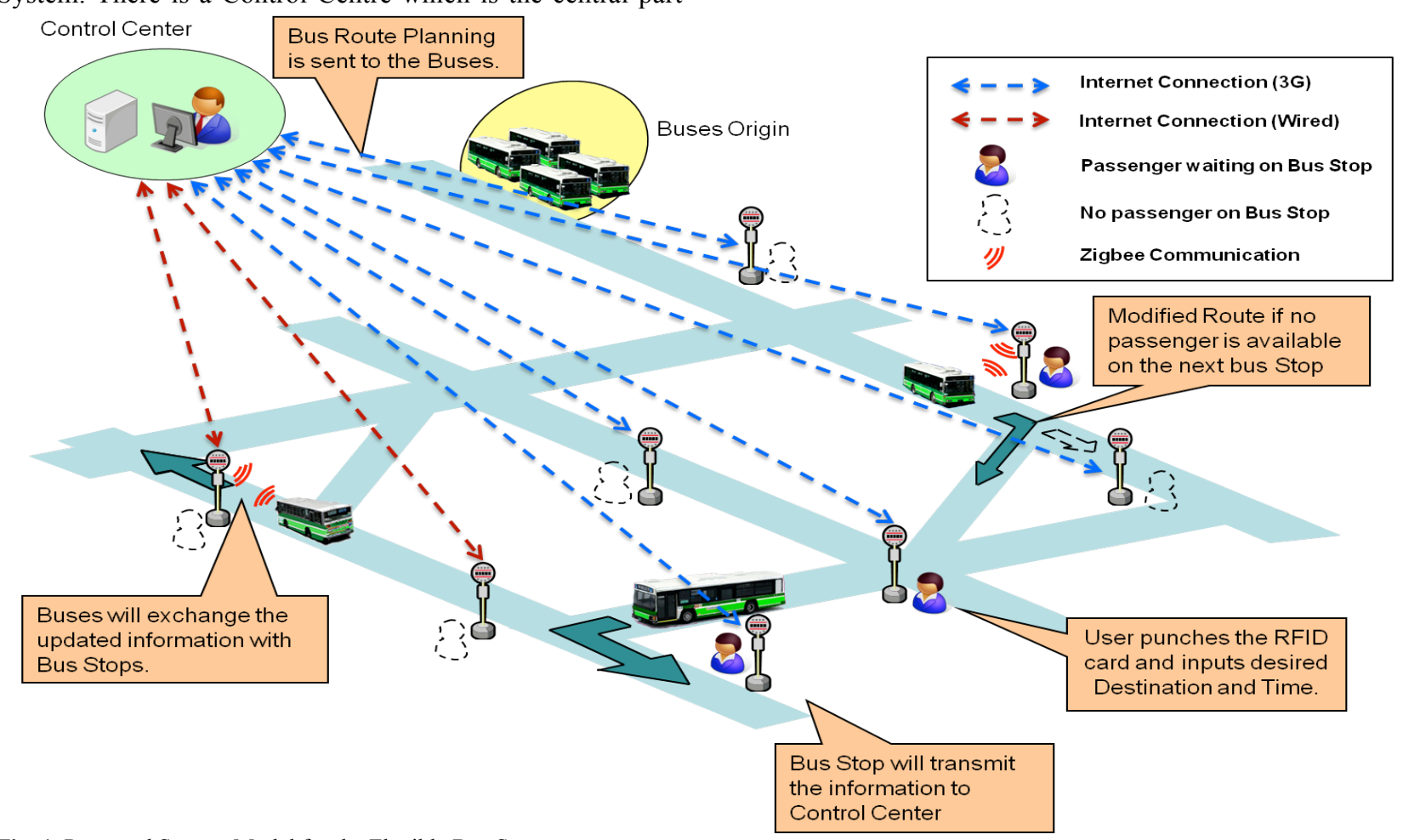

Fig. 1. Proposed System Model for the Flexible Bus Systems

The Control Centre is connected to all the Bus Stops through the internet (Wired or Wireless). We are considering Wired and Wireless both because if the system is to be deployed in the city, wired internet is easily available but if the system is to be deployed in rural areas, providing wired internet facility will be very difficult because of the cost, so we are considering wireless internet in these cases. Similarly if wireless internet is not available in some areas, wired Internet can be used. When we talk about wireless internet, $3 \mathrm{G}$ is the most convenient option because it can give long range communications. In some parts of the world, licensing can be a problem for $3 \mathrm{G}$; in that case wired internet can be used.

An interesting feature of this research is that Buses and Bus Stops communicate with each other using short range wireless technology; "ZigBee" so that all the information between Buses and Bus Stops is transferred using "ZigBee".

One important thing to note here is that Bus and Control Centre are not connected to each other directly, they communicate to each other through Bus Stop, means the information from Control Centre is first transferred to Bus Stop and then from Bus Stop to Bus vice versa. This way there will be no need of $3 \mathrm{G}$ module installation in Buses which will definitely be more than 1 in most of the cases. This will greatly reduce the total cost of the system.

\section{Intelligent Bus Stops}

The Bus Stops in this system are very smart and we call them "Intelligent Bus Stops". The passengers carry RFID of the system and carrying all the information about the system e.g. no. of passengers on the Bus Stops, Bus locations, passenger ids, Bus Stops ids etc. The Control Centre performs all the actions like passenger identification, sending route information to Buses through Bus Stops and sending Bus locations to Bus Stops etc. card which contains all the information about the passenger. Whenever a passenger goes to the Bus Stop, he/she will punch his/her RFID card to those RFID card readers installed at Bus Stop and gets recognized by the system. After getting recognized by the system, the passenger can enter the destination and will get the response from the system e.g. which Bus to ride and how much is the wait time.

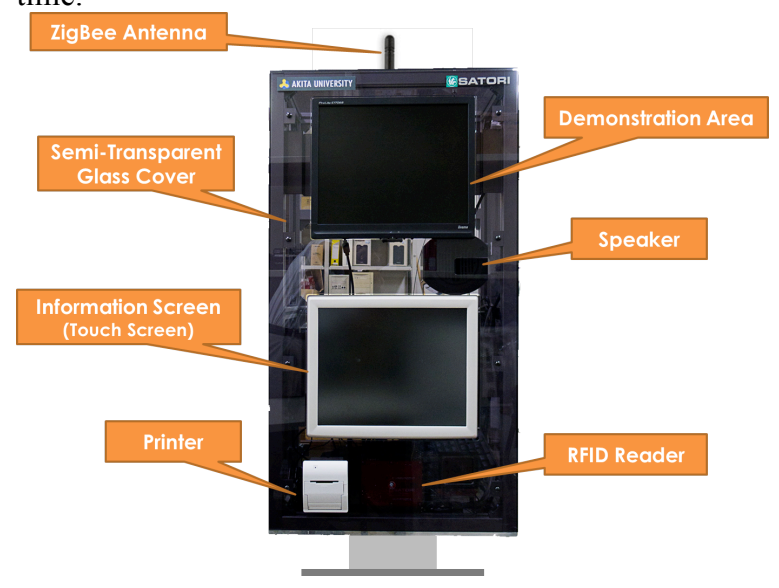

Fig. 2. Prototype of developed Bus Stop for the Flexible Bus Systems

Below are the details of different components of Prototype Bus Stop shown in Figure 2. 


\subsection{ZigBee Antenna}

The information between Buses and Bus Stops will be transferred through a low power and low cost wireless technology, ZigBee. The Antenna is placed at a certain height on the Bus Stop so that maximum clear light of sight is achieved while communication between Bus and the Bus Stops. The operation of ZigBee and the type of data that is going to be transferred through and from Buses and Bus Stops are beyond the scope of this paper.

\subsection{Demonstration Area}

The screen called Demonstration Area displays a demo of how to use the system. A repeated video file will keep playing on this screen to help passenger operate the system. Furthermore this display will also show important details about the selected destination of the passenger like maps etc.

\subsection{Semi Transparent Glass Cover}

The whole front of the prototype bus stop is covered with a semi-transparent glass cover which will protect its internal component from outside exposure.

\subsection{Speaker}

The system is designed in a way that it helps the passenger to navigate through the screens by producing user friendly audio messages. For example, when a passenger arrives at the bus stop to select his/her destination, the system welcomes the passenger by playing a welcome message and some start-up instructions like "Welcome to the Flexible Bus Systems."

\subsection{Information Screen}

Information Screen is the most important component of the proposed Intelligent Bus Stop. This is where all magic happens. Information Screen is a touch screen. A Passenger can touch the screen and different actions are performed. A user friendly interface specially designed for Intelligent Bus Stop will be displayed to the passenger with different options to select from, which will help passenger in selection of his/her destination.

\subsection{Printer}

A printer is also installed in the intelligent bus stop. A passenger can get the information from the system and can get the copy of that information using the printer. The System will print the required information e.g. which bus to ride, arrival time of the bus, and time to destination and price for the ticket.

\subsection{RFID Reader}

An RFID reader is also installed within the intelligent bus stop. Each registered member of the Flexible Bus Systems carries a RFID card which contains all the information of the passenger. Whenever a passenger arrives at the bus stop and swipes his/her RFID card against the RFID card reader installed at the bus stop, a personalized home page of the passenger will be displayed to him which will help the passenger in selecting his destination. More details about selecting destination from different options later in the paper.

\section{Passenger Interaction at the Bus Stop}

The main idea of this System is to make passenger comfortable by providing him more and more information.
The equipment at the Bus Stops is designed in such a way that passengers can use them easily. The Passengers in FBS are categorized as

Members: The members are the passengers who are registered with FBS. Each member carries an ID card; we call it "FBS ID Card".

Non-Members: The non-members are the passengers who are not registered with FBS and are not carrying "FBS ID Card", e.g. One-Time-Riders or Tourists (more details in upcoming section).

Whenever a passenger arrives at the Bus Stop, he is presented with a beautiful graphical user interface asking him to Swipe his FBS Passenger card (RFID Card) against the RFID card reader. After swiping the card, the Bus Stop will send this information to the Control Centre using the Internet (since Bus Stops and Control centre are connected to each other through the internet, Wired or Wireless.) to check for the validation. The Control centre checks the information into its database and if the Passenger is not registered with the system, the Control Centre sends an error message to the Bus Stop which displays the information to the passenger on the Touch Screen asking him to contact the registration office for further assistance. If however Control Centre authenticates the passenger id it sends the Name of the passenger to the Bus Stop. The Bus Stop after getting the authentication from the control centre about the passenger will present the Passenger with a Menu. The screens shown to the passenger are very easy to use and are selfexplanatory. For the ease of the passengers, screens are bilingual (Japanese, English). Below are the options to be presented to the Passenger.

\subsection{Direct Input}

This option allows the passengers to enter their destination directly by showing them on-screen keyboard. The system is designed using 'Instant Search' (Instead of a user having to wait until they've clicked the "search" button, results begin to show up as they type in the search box) which will help passengers in finding their desired input. After getting the desired destination, the passenger can tap "Select as Destination" button to select the desired destination as a final destination. As discussed earlier, passengers can also enter the telephone no. if they don't know the exact address of their destination, and FBS will show them the nearest Bus Stop where the Bus will drop them.

\subsection{Nearby Places (Frequently visited places)}

FBS is a smart system; it remembers the places passengers normally visit like Hospitals, City Office, and Police Stations etc. By using this option, the passengers don't need to add the destination on their own (No direct input is required as discussed in A), they can select from the listed options which will save time and is less hassle free. Each Intelligent Bus Stop has its corresponding nearby places, for example "BUS STOP A" can have "City Office" as a nearby place whereas "BUS STOP B" can have "Central Hospital" as a nearby place.

After passenger has selected his desired destination, he is shown the details of which Bus to ride along with the arrival time of the Bus on current Bus Stop. Passenger is also shown a Mini map which displays the current location of the Bus. Passenger is then asked whether he wants to ride this Bus or not. More details on the role of Control Centre in 
deciding which Bus a particular passenger should ride and arrival time of the Bus on current Bus Stop in upcoming sections.

\subsection{Choose from History}

In addition to remembering the frequently visited places, FBS also remembers the history of each member. Each time a passenger rides a bus, FBS saves this information into its databases, so next time whenever a passenger comes to the Bus Stop to ride a Bus it shows passenger his last four recent visits. A passenger can quickly select one from these four choices if he wants to visit the same place again. This option is very useful for regular riders like school or college students, workmen, or old age citizens who have to visit hospitals for their regular check-ups.

\subsection{Famous Places}

Each city has its own attractions and famous places; The Flexible Bus System is very convenient in a sense that it can be used as a Search Engine for searching nearby famous places like restaurants, famous parks and other famous cultural places. For example if a passenger doesn't know much about the city and he wants to go out for some famous parks, he can use this option and The Flexible Bus System will suggest him nearby famous parks and also guides him about which Bus to ride along with the distance to destination and approximate total ride time. This option can be really helpful for tourists who visit for sightseeing etc.

\subsection{Tourist / Non-Members}

The Flexible Bus System is not only convenient for regular bus riders who are registered to The Flexible Bus System (carrying FBS ID Card) but it is also very helpful for the non-members (who are not carrying FBS Passenger Card) like Tourists or one-time riders. If a tourist wants to ride the Bus, he can choose the Option "Tourist/Non Members", the Flexible Bus System will show him option D (Famous Places) as discussed above. This option contains all the information like screenshots, history etc. about these famous places. The Tourist can go through these details and decides where to go. Since Tourists are not registered with FBS, their options of interacting with the System are limited, like if a rider is not carrying a FBS ID Card it's not possible to save/show his/her riding history next time he/she comes to the Intelligent Bus Stop. Similarly E-ticketing option won't be available for non-members until they register their Bank Accounts with FBS, for which they need to become a member of FBS.

\section{Information Flow Between Control Centre and Bus Stop}

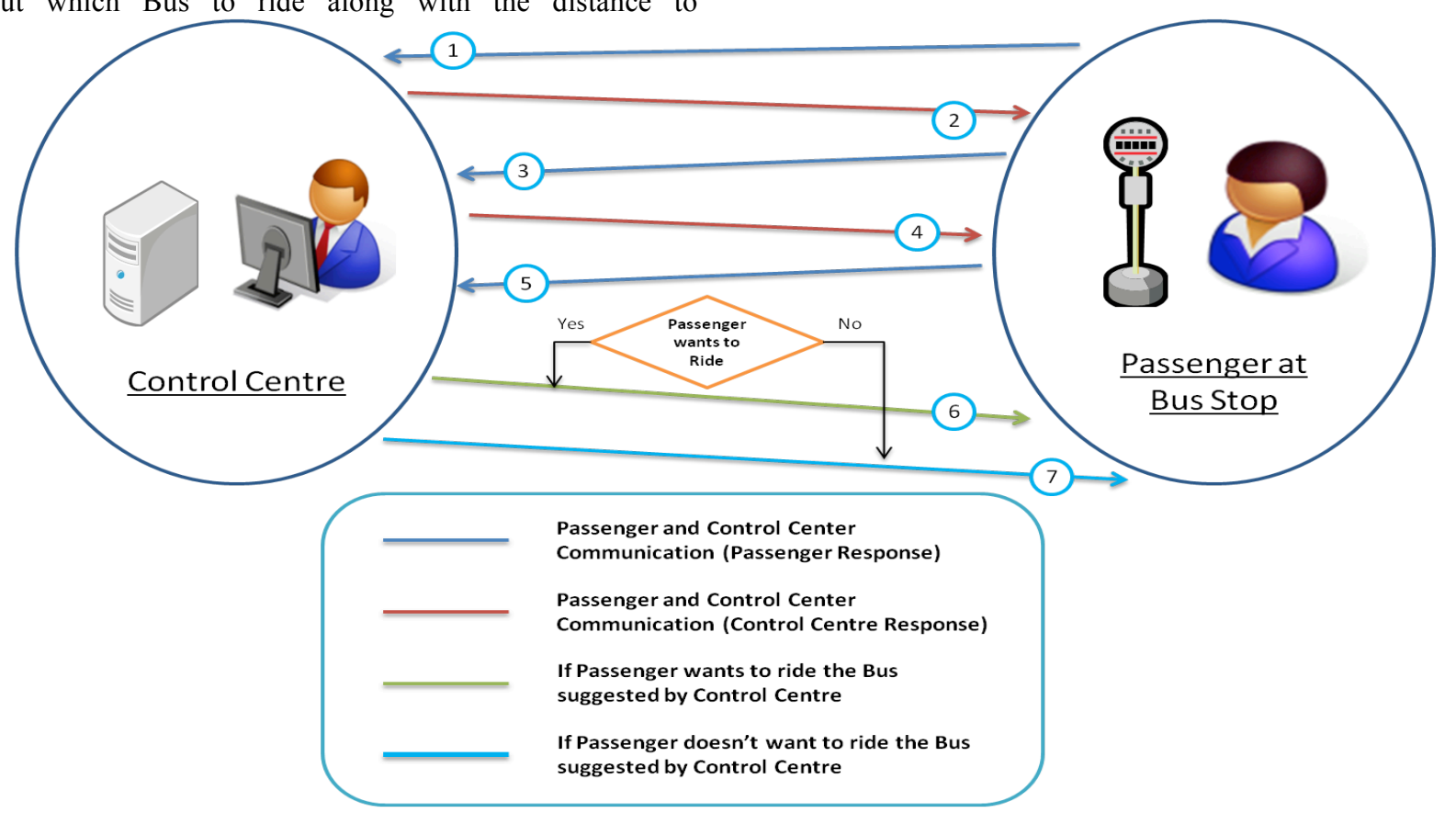

Fig. 3. Information flow between Control Centre and Bus Stop

The Flexible Bus System is an automated System. All the information is in the digital form. The information between Control Centre and Bus Stops as discussed in previous section (Passenger Interaction at Bus Stop) is transferred using High Speed internet.

Figure 3 shows the flow of information between the Intelligent Bus Stop and the Control Centre when a passenger arrives at the Bus Stop and starts interacting with the System

1. When a Passenger punches his FBS ID Card against the Sensor, the Passenger ID is transferred to the Control Centre from Bus Stop.

2. Control Centre checks its database for Passenger ID to make sure if this particular ID is registered or not. If the
ID is registered, the Control Centre will send the Name of the Passenger corresponding to that ID to the Bus Stop.

3. The Passenger will be shown an authentication successful message and a Menu is shown to select the destination out of 4 choices as discussed in section "Passenger Interaction at Bus Stop". Passenger's choice is then sent to the Control Centre.

4. The Control Centre after receiving Passenger's Choice, checks for the location of the buses. After Control Centre gets the location of the nearby Bus, the passenger on the Bus Stop is shown a screen which displays Bus (Bus No.) to ride, Bus's current location, and arrival time of that particular Bus on that particular Bus Stop. 
5. The passenger is then given a choice whether he wants to ride this particular Bus or Not and that choice is sent to the Control Centre.

6. If the Passenger chooses YES, the Control Centre asks the nearby Bus to pick up the passenger.

7. If the Passenger chooses NO, the proceedings for the passenger are cancelled.
Figure 4 shows the flow of information between the Intelligent Bus Stop and the Control Centre when a passenger arrives at the Bus Stop and starts interacting with the System in an easy to understand flow chart.

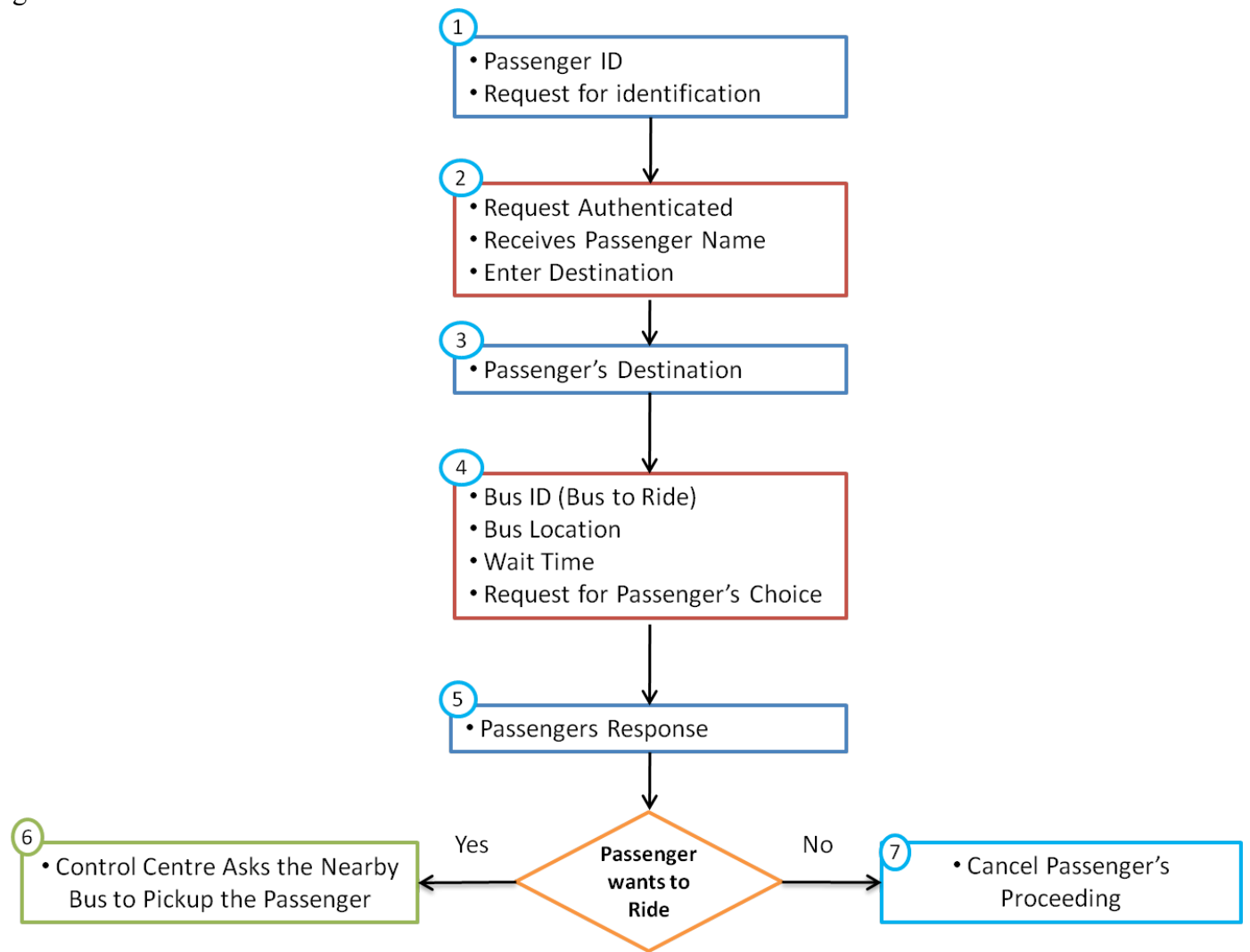

Fig. 4. Flow chart for information flow between Control Centre and Bus Stop

4. No. of passengers waiting on the Bus Stop.

The Control Centre's decision to ask a Bus to pick up a particular passenger on a particular Bus Stop depends upon the following facts:

1. Bus is near that particular Bus Stop where passenger is waiting.

2. Bus has seats available.

3. The current route of the Bus.
The whole Flexible Bus System is designed in a way that passenger doesn't have to wait for long at the Bus Stops. The Intelligent Bus Stops make this possible using fast data transfer between the Control Centre and the Bus Stops using high speed internet. The flow shown above takes a mere few seconds to respond to the passenger's response.

\section{Role of Control Centre in the Flexible Bus Systems}




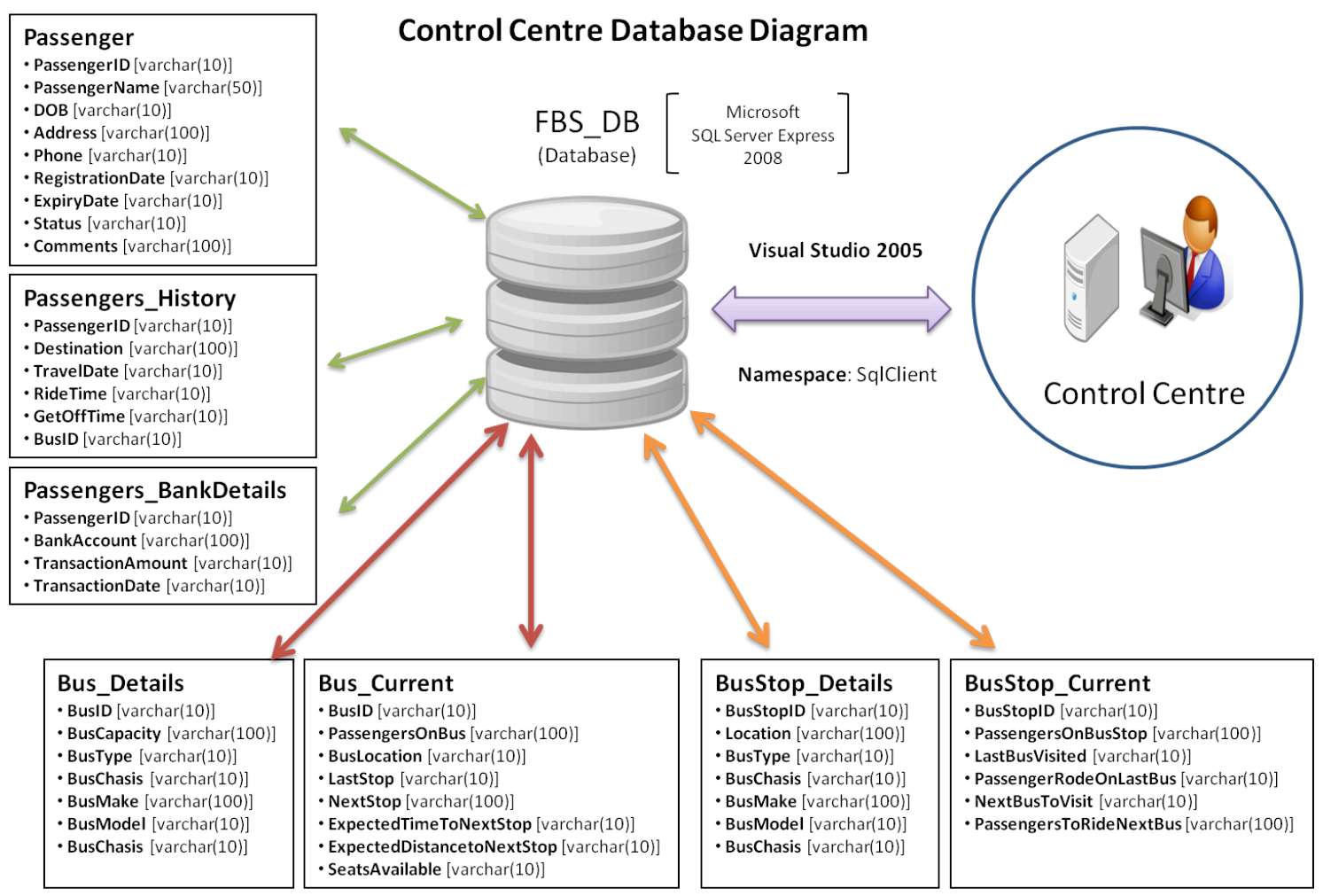

Fig. 5. Control Centre role in the Flexible Bus Systems

The Control Centre is the brain of the Flexible Bus Systems. It contains all the information about the Buses, Bus Stops and Passengers etc. The Control Centre is very smart in a sense that it takes instant decisions about the Buses and the Passengers depending upon their current locations.

Figure 5 shows the role of the Control Centre whenever a passenger arrives at the Bus Stop and starts interacting with the System. The Control Centre has a Database which contains all the information about the Passengers as shown above. We have used Microsoft SQL Server Express 2008 as a Database tool and Visual Studio 2005 as a tool for interacting with the Database.

Since this paper discusses the Intelligent Bus Stops so we are not going into details of How Control Centre checks the information? Similarly role of the Control Centre with respect to Buses is also beyond the scope of this paper.

\section{Conclusions}

The Flexible Bus Systems is an efficient and a smart Demand Responsive Transit (DRT). It is flexible in a sense that it can change dynamically according to the demands of the passengers. The system can fulfil the demands of the passengers in a way that they have to wait less on the Bus Stops and even if they miss the Bus they can be entertained by the next bus without waiting for very long.
The Flexible Bus System is designed to make passengers comfortable while travelling through Buses. Passenger interaction with the System starts when passenger arrives at the Bus Stops, so intelligent Bus Stops are the central part of this System. Intelligent Bus Stops are designed in a way that they are bilingual (Japanese, English). The screens of this System are very easy to use and are self-explanatory just like Bank ATM (Automated Teller Machine). The registered members of the System are given different choices to select their destinations, for example, direct input, nearby places, history and famous places. Non-members like one-timeriders or Tourists can also use this system but with limited choices.

The intelligent Bus Stops can act as a Search Engine for different places, like restaurants, hospitals and famous places etc. Even if the passengers are not aware of the exact location of their destination or exact Bus Stop No. the system can guide them to find their destination place.

The Flexible Bus System is automated which makes it fast and efficient. Passenger inputs are responded instantly which makes this system very smart.

\section{Acknowledgements}

This work is supported by the Strategic Information and Communication R\&D Promotion Programme 102302005 from the Japanese Ministry of Internal Affairs and Communications.

\section{References}

[1] Jin \& Huang (2009) “An Intelligent Model for Urban Demandresponsive Transport System Control”, Journal of Software, Vol. 4, No. 6.

[2] Leitao \& Ferreira (2000) "Graphical Control of Autonomous, Virtual Vehicles", Proceedings of the IEEE Vehicular Technology Conference, Tokyo, Japan, pp. 507-511.
[3] Lee, Ryu, \& Paik (2008) "A Concept for Ubiquitous Transportation Systems and Related Development Methodology", International IEEE Conference on Intelligent Transportation Systems, pp.37-42.

[4] Wahle \& Scbreckenberg (2001) "A Multi-Agent System for On-lie Simulations based on Real-World Traffic Data", Proceedings of the International Conference on System Sciences, Hawaii, USA, pp. 1125-1133. 
[5] Utsumi, Konno, Kanno, Yukimatsu, Kobayashi \& Hashimoto (2009) "A Feasibility study on the Buffer-Less Routing Networks using Deflection Routing Control”, Proceedings of IEICE Vol. J92B, No. 11 pp. 1741-1749.

[6] Julstrom \& Raidl (2002) "Initialization is robust in evolutionary algorithms that encode spanning trees as sets of edges", Symposium on Applied Computing, pp. 547-552.

[7] Iqbal, Yukimatsu \& Ichikawa (2011) "A Research on the Flexible Bus Systems Using Zigbee as a Communication Medium”,
Proceedings of 4th IFIP International Conference on New Technologies, Mobility and Security, Paris, France, pp. 1-5.

[8] Perkins \& Bhagwat, (1994) "Highly Dynamic DestinationSequenced Distance-Vector Routing (DSDV) for Mobile Computers", Proceedings of ACM SIGCOMM'94 Conference on Communications Architectures, Protocols and Applications, pp. 234-244. 